\title{
Natural Deduction System for the Logic of Binary Relations Based on the Algebraic Tradition*
}

\author{
Márcia R. Cerioli ${ }^{1}$, Leandro Suguitani ${ }^{2}$, Petrucio Viana ${ }^{3}$ \\ ${ }^{1}$ Instituto de Matemática \\ Programa de Engenharia de Sistemas e Computação-COPPE \\ Universidade Federal do Rio de Janeiro (UFRJ) - Rio de Janeiro - RJ - Brazil \\ ${ }^{2}$ Instituto de Matemática e Estatística \\ Universidade Federal da Bahia (UFBA) - Salvador - BA - Brazil \\ ${ }^{3}$ Instituto de Matemática e Estatística \\ Universidade Federal Fluminense (UFF) - Niterói - RJ - Brazil \\ marcia@cos.ufrj.br, leandro.suguitani@ufba.br, petrucio_viana@id.uff.br
}

\begin{abstract}
We present a proper natural deduction system for a logic of binary relations based on the algebraic tradition. Our system is an evolution from 1975 W.W. Wadge's formalism. We point out some aspects where Wadge's system fails to fit nowadays standards of natural deduction system and we fix it.
\end{abstract}

\section{Introduction}

Roughly speaking, a relational calculus is a formal system in which "information" is expressed in terms of properties of relations and "reasoning" is performed through reasoning on relations. Several relational calculi have been introduced for many purposes and with different approaches. One of the most prominent is the De Morgan-Peirce-Schröder Calculus of Binary Relations (CBR) [De Morgan 1860, Peirce 1883, Schröder 1895]. This calculus deals, basically, with equalities between terms built, as usual, by iterated aplications of the binary operators union $(+)$ and composition $(;)$, and the unary operators complementation $\left({ }^{-}\right)$and reversion $\left({ }^{-}\right)$on the variables $x_{1}, x_{2}, \ldots, x_{n}, \ldots$ and the constant identity ( $1^{\prime}$ ). The names of the operators suggests their intuitive meaning. For the purposes of this investigation, CBR can be defined as the set of all equalities which are true when-for every universe of discourse $U$ - the variables range on all the binary relations on $U$, and the operators are interpreted as usual, according to their names.

A central problem-which motivated much of the development and interest in the relational calculus - was to present an equational axiomatization for CBR. This problem was raised by A. Tarski in [Tarski 1941]. Later on, B. Jonsson and A. Tarski introduced an algebraic theory called relation algebra (RA) [Jónsson and Tarski 1948] whose set of axioms was proposed as a solution for it and, even though not explicitly, it is very close to the axiomatization presented below. An algebraic structure $\mathfrak{A}=\left\langle A,+, ;,{ }^{-},{ }^{\smile}, 1^{\prime}\right\rangle$, where $A$ is a non-empty set, + and ; are binary operations on $A,{ }^{-}$and ${ }^{-}$are unary operations on $A$, and $1^{\prime}$ is a distinguished element of $A$, is a relation algebra iff the

\footnotetext{
${ }^{*}$ We thank the anonymous reviewers for their valuable comments and suggestions which contributed to the improvement of this paper.
} 
following axioms hold, for all $a, b, c \in A$ :

$\begin{array}{llll}\text { (RA1) } & a+(b+c)=(a+b)+c & (\mathrm{RA}) & a^{\smile}=a \\ \text { (RA2) } & a+b=b+a & \text { (RA7) } & (a ; b)^{\smile}=b^{\smile} ; a^{\smile} \\ \text { (RA3) } & \left(a^{-}+b\right)^{-}+\left(a^{-}+b^{-}\right)^{-}=a & \text { (RA8) } & (a+b) ; c=a ; c+b ; c \\ \text { (RA4) } & a ;(b ; c)=(a ; b) ; c & \text { (RA9) } & (a+b)^{\smile}=a^{\smile}+b^{\smile} \\ \text { (RA5) } & a ; 1^{\prime}=a & \text { (RA10) } & \left(a^{\smile} ;(a ; b)^{-}\right)+b^{-}=b^{-}\end{array}$

The standard models for these axioms are structures where the domain $(A)$ is a set of (binary) sub-relations of an equivalence relation, closed under the operations of union $(+)$, composition $(;)$, complementation $\left(^{-}\right)$, reversion $\left(^{-}\right)$, and having the identity relation $\left(1^{\prime}\right)$ as a distinguished element of the domain. These models are usually called the $a l$ gebras of binary relations. A relation algebra is representable if it is isomorphic to an algebra of binary relations. R. Lyndon constructed a relation algebra which is not representable [Lyndon 1950], showing that the above set of axioms is incomplete for the class of the algebras of binary relations, meaning that there are some equations which hold for every algebra of binary relations but cannot be proved from these axioms by means of 'algebraic methods'. Tarski proved that the class of representable relation algebras is, indeed, axiomatizable by a set of equations [Tarski 1955], but his work does not exhibit such a set. It was Lyndon who presented a set of axioms which is complete [Lyndon 1956], but this set is infinite and hugely complex to be here described. More generally, R. D. Monk proved that the set of equations true in all algebras of binary relations is not finitely axiomatizable [Monk 1964], B. Jonsson proved that this set cannot be axiomatized by a set of equations using finitely many variables [Jónsson 1991], and H. Andréka proved that in any equational axiomatization of these equations, for any $k \in \mathbb{N}$, there are infinitely many equations containing more than $k$ variables, and containing at the same time ; , , and one of,$+ \cdot$ (wich is definable) [Andréka 1997]. It follows from these results that the tentatives of providing CBR with an equational set of axioms take us to either an incomplete set of axioms or a very complex one. An immediate route for avoiding this problem would be taking CBR as the set of equations provable in a first-order logic with equality extended with axioms defining the CBR-operators, as it had been already noticed by both Peirce and Schröder. However, this "radical" turn would make us loose all the algebraic flavor and insights that comes with it over binary relations. In other words, we would be throwing the baby out with the bath water if we just gave up of the relational calculus as it was originally conceived. Thus, some effort has been made in order to keep the operations on binary relations as they were algebraically introduced and still searching for alternatives to have a complete and "friendly" framework to manipulate them. In this direction, some "wider" logical formalism have been proposed. Usually, this is done by extending the language through the inclusion of variables for individuals and extending the inference mechanism by adapting the usual logical machinery which takes advantage of this second type of variable. This has been done for natural deduction [Wadge 1975], (dual) semantical tableaux [Orlowska 1991], and sequent calculus [Maddux 1983].

In this paper we present a natural deduction system for CBR which is an improvement of the above mentioned Wadge's system. We have chosen this system due to some formal aspects of its presentation which fail to fit nowadays standards of natural deduction systems. In Section 2, we revisited Wadge's original system to show how our system W, introduced in Section 3, improves it. In Section 4 we make some considerantions. 


\section{Wadge's Original System}

Despite not having a precise definition of natural deduction system (ND-system), it has been accepted that such systems have many distinguishing features which split them apart from other kind of logical formalisms (cf. [Indrzejczak 2010] and the bibliography therein). We will show that Wadge's system fails to fulfill such features and, therefore, needs to be fixed in order to be presented as a proper ND-system. We recognize that our criticisms of Wadge's formalism may be somewhat anachronistic, since our analysis is based on some formal refinements which were developed much later than Wadge's work came out. But still this should not be a reason to not make the system better, specially considering modern developments of ND-systems with respect to formal aspects of it.

In Wadge's system, formulas are expressions of the form $x R y$, where $x$ and $y$ are point variables and $R$ is a term defined recursively, as usual, from the constants $\Omega$, $U$ and $E$ - to denote, respectively, the binary relations empty, universal and identity, respectively - by applying the syntactical operators $\cup, \cap, ;,-$ and ${ }^{\cup}$ - to denote, respectively, the operations of union, intersection, composition, complementation, and reversion on relations. The proof system is defined by the following inference rules, where $F$ and $G$ are arbitrary formulas; $x, y$ and $v$ are arbitrary variables; $R$ and $S$ are arbitrary terms; $\Gamma$ is an arbitrary set of formulas; in $(; \mathrm{E}), v$ is a new variable; and in $(\subseteq \underline{\mathrm{I}}), x$ and $y$ do not occur in any formula in $\Gamma$ :

$$
\begin{aligned}
& \frac{\Gamma \vdash F}{\Gamma, G \vdash F}(\mathrm{AI}) \quad \frac{\Gamma \vdash G \quad \Gamma, G \vdash F}{\Gamma \vdash F}(\mathrm{AE}) \\
& \frac{x R y}{x R \cup S y} \frac{x S y}{x R \cup S y}(\cup \mathrm{I}) \quad \frac{\Gamma, x R y \vdash F \quad \Gamma, x S y \vdash F \quad \Gamma \vdash x R \cup S y}{\Gamma \vdash F}(\cup \mathrm{E}) \\
& \frac{x R y x S y}{x R \cap S y}(\cap \mathrm{I}) \quad \frac{x R \cap S y}{x R y} \frac{x R \cap S y}{x S y}(\cap \mathrm{E}) \\
& \frac{\Gamma, x R y \vdash a \Omega a}{\Gamma \vdash x \bar{R} y}\left({ }^{-} \mathrm{I}\right) \quad \frac{\Gamma, x R y \vdash F \Gamma, x \bar{R} y \vdash F}{\Gamma \vdash F}\left({ }^{-} \mathrm{E}\right) \\
& \frac{x R y x \bar{R} y}{a \Omega a}(\Omega \mathrm{I}) \quad \frac{a \Omega a}{F}(\Omega \mathrm{E}) \\
& \frac{\Gamma, x U y \vdash F}{\Gamma U y}(U \mathrm{I}) \quad(U \mathrm{E}) \\
& \frac{\Gamma \vdash x R y}{\Gamma \vdash y \breve{R} x}(\mathrm{I}) \quad \frac{\Gamma \vdash x \breve{R} y}{\Gamma \vdash y R x}(\breve{E}) \\
& \frac{x R v v S y}{x R ; S y}(; \mathrm{I}) \quad \frac{\Gamma, x R v, v S y \vdash F \Gamma \vdash x R ; S y}{\Gamma \vdash F}(; \mathrm{E}) \\
& \frac{\Gamma, x R y \vdash x S y}{\Gamma \vdash R \subseteq S}(\subseteq \mathrm{I}) \quad \frac{R \subseteq S x R y}{x S y}(\subseteq \mathrm{E})
\end{aligned}
$$




$$
\frac{}{x E x}(E \mathrm{I}) \quad \frac{x R v v E y}{x R y}(E \mathrm{E})
$$

According to A. Indrzejczak, an ND-system may be categorized according to its "basic items (data structure) on which inference rule are defined", which can be formulas, sets of formulas, ordered pairs of formulas, formulas with labels, sequents etc. Taking Indrzejczak's viewpoint into consideration, the first aspect of this system that we have fixed is that Wadge has introduces rules which are applied, at least, on two different kind of data structures. In fact, some rules as $(\cup \mathrm{I})$ are applicable on formulas $(x R y, x S y)$, whereas other as $(\cup \mathrm{E})$ are applicable on derivations $(x R y \vdash F, x R y \vdash F)$. All our rules apply on sequents $(\Gamma \vdash x R y)$. Moreover, it seems agreedable in the literature that inference rules in a ND-system is about introducing and eliminating logical operators. However, rules ( $U E$ ) and (I) do not follow this "criteria". Actually, these rules would be expected to be a structural property of proofs and not inference rules of the system.

Now let us have a look on rule ( $\mathrm{E})$. At first sight it seems to be a proper inference rule since it eliminates an operator $\left(^{-}\right)$. Nonetheless, this rule brings up a different data structure for the system. If we assume formulas as the data structures (based, for instance, on rules $(\cup \mathrm{I})$ and $(\cap \mathrm{I})$ ), then $(\mathrm{E})$ cannot be an inference rule since the objects of this rule are not formulas. On the other hand, if we assume that rule $(\mathrm{E})$ is applied on sequents (or derivations) as data structures, then the notion of proof in this system should be revisited since it is defined as a linear sequence of formulas (cf.[Wadge 1975], p. 4, 6-8). Moreover, Indrzejczak pertinently points out that an inference rule over a sequent in a ND-system should introduce or eliminate operators only in the consequent (second term) of the sequents. So from this point of view, even if we consider sequents as data structures of this system, the rule $(\mathrm{E})$ is not properly presented as an inference rule of a ND-system. Lastly, in ND-systems, it is not typical to have elimination and introduction rules for operators which do not belong to the object language as Wadge's does in rules $(\subseteq \mathrm{I})$ and $(\subseteq \mathrm{E})$ since the symbol $\subseteq$ is an abbreviation for a formula and not an operator of the language itself.

\section{The System W}

In this section, we introduce the ND-system W (in honor of Wadge's pioneering system). The main difference between $W$ and Wadge's original system is in the mechanism of inference, where we substitute and adapt some of the original rules in order to organize the formalism as a proper ND-system. Regarding the language, we just change the symbols for a more up to date notation, keeping the same semantics. We presented our rules following the style of presentation for natural deduction adopted in [Sundholm 1991].

\subsection{Syntax}

In this section we define the language - vocabulary, terms, and formulas-of the system $\mathrm{W}$.

The W-vocabulary consists of an enumerable sequence of symbols for individuals $\left\langle x_{1}, x_{2}, \ldots, x_{i}, \ldots\right\rangle ;$ an enumerable sequence symbols for relations $\left\langle R_{1}, R_{2}, \ldots, R_{i}, \ldots\right\rangle$; a set of symbols for operations with three binary operators $\langle+, \cdot, ;\rangle$, two unary operators $\left\langle^{-},-\right\rangle$and two zero-ary operators $\left\langle 0,1,1^{\prime}\right\rangle$; 
and parentheses as auxiliary symbols. The set of symbols for individuals and symbols for relations are denoted by Syml and SymR, respectivelly, and their elements are denoted generically by the letters $x, y, z, u, v, w$ and $R, S, Q$ (indexed or not). The terms of W, W-terms, are defined inductively by the following rules of term construction: $T::=R|0| 1\left|1^{\prime}\right| T^{-}\left|T^{\smile}\right| T_{1}+T_{2}\left|T_{1} \cdot T_{2}\right| T_{1} ; T_{2}$, where $R \in$ SymR. The set of $\mathrm{W}$-term is denoted by TerW and its elements are denoted generically by the letters $T, U, V$ (indexed or not). The formulas of $\mathrm{W}, \mathrm{W}$-formulas, are expression of the form: $x T y$, where $x, y \in \mathrm{Syml}$ and $T \in \mathrm{TrmW}$. The set of $\mathrm{W}$-formulas is denoted by FrmW and its elements are denoted generically by the letters $\varphi, \psi, \theta$ (indexed or not).

\subsection{Semantics}

In this section we define a semantics-structures of interpretation; denotation of terms; satisfaction, truth and validity of formulas; and semantical consequence of a formula from a set of formulas-for the language of $\mathrm{W}$.

A structure for $\mathrm{W}, \mathrm{W}$-structure, is an ordered pair $\mathfrak{S}=\langle\mathrm{U}, \mathrm{I}\rangle$, where $\mathrm{U}$ is a non empty set, called the universe of discourse of $\mathfrak{S}$; I is a function, I : SymR $\rightarrow \wp(\mathrm{U} \times \mathrm{U})$, mapping symbols for relations to binary relations on $\mathrm{U}$ called the interpretation function of $\mathfrak{S}$. The denotation of a W-term $T$ in a $\mathrm{W}$-structure $\mathfrak{S}$ is the binary relation $\llbracket T \rrbracket_{\mathfrak{S}}$ on $\mathrm{U}$, defined recursively by the following rules of formulas denotation:

$$
\begin{aligned}
\llbracket R \rrbracket_{\mathfrak{S}} & ::=\mathrm{I}(R), \text { for all } R \in \mathrm{Sym}_{\mathrm{R}} & \\
\llbracket 0 \rrbracket_{\mathfrak{S}} & ::=\emptyset & \text { (empty) } \\
\llbracket 1 \rrbracket_{\mathfrak{S}} & ::=U \times U & \text { (universal) } \\
\llbracket 1^{\prime} \rrbracket_{\mathfrak{S}} & ::=\{(a, a): a \in U\} & \text { (identity) } \\
\llbracket T^{-} \rrbracket_{\mathfrak{S}} & ::=\llbracket T \rrbracket_{\mathfrak{S}}{ }^{c} & \text { (complementation) } \\
\llbracket T \rrbracket_{\mathfrak{S}} & ::=\llbracket T \rrbracket_{\mathfrak{S}}{ }^{-1} & \text { (reversion) } \\
\llbracket T+U \rrbracket_{\mathfrak{S}} & ::=\llbracket T \rrbracket_{\mathfrak{S}} \cup \llbracket U \rrbracket_{\mathfrak{S}} & \text { (union) } \\
\llbracket T \cdot U \rrbracket_{\mathfrak{S}} & ::=\llbracket T \rrbracket_{\mathfrak{S}} \cap \llbracket U \rrbracket_{\mathfrak{S}} & \text { (intersection) } \\
\llbracket T ; U \rrbracket_{\mathfrak{S}} & ::=\llbracket T \rrbracket_{\mathfrak{S}} \circ \llbracket U \rrbracket_{\mathfrak{S}} & \text { (composition) }
\end{aligned}
$$

An assignment in a $\mathbf{W}$-structure $\mathfrak{S}, \mathfrak{S}$-assignment, is a function a : Syml $\rightarrow U$, mapping symbols for individuals to elements of $U$. Given a W-formula $x T y$, a W-structure $\mathfrak{S}$, and a $\mathfrak{S}$-assignment $\mathrm{a}$, we say that: denoted by $\mathfrak{S} \vDash x T y$, when $\mathfrak{S}, \mathrm{a} \vDash x T y$, for every $\mathfrak{S}$ assignment a; $\mathfrak{S}$ and a satisfy $x T y$, denoted by $\mathfrak{S}, a \vDash x T y$, when $(\mathrm{a} x, \mathrm{a} y) \in \llbracket T \rrbracket_{\mathfrak{A}} ; x T y$ is true in $\mathfrak{S}$ (alternatively, $\mathfrak{S}$ is a model of $x T y$ ), denoted by $\mathfrak{S} \vDash x T y$, when $\mathfrak{S}$, a $\vDash x T y$, for every $\mathfrak{S}$-assignment a; $x T y$ is valid, denoted by $\vDash x T y$, when $\mathfrak{S} \vDash x T y$, for every $\mathrm{W}$-structure $\mathfrak{S}$. Given a set of $\mathbf{W}$-formulas $\Gamma$, we write $\mathfrak{S}$, $a \vDash \Gamma$ when $\mathfrak{S}$, a $\vDash_{W} \gamma$, for every $\gamma \in \Gamma$. And we say that a $W$-formula $\varphi$ is a (local) semantical consequence of $\Gamma$ in W ( $\varphi$ is a semantical consequence of $\Gamma$ ), denoted by $\Gamma \vDash \varphi$, when, for all W-structure $\mathfrak{S}$ and $\mathfrak{S}$-assignment $a$, if $\mathfrak{S}, \mathrm{a} \vDash \Gamma$ then $\mathfrak{S}, \mathrm{a} \vDash \varphi$.

\subsection{Mechanism of inference}

In this section we define a natural deduction system-introduction and elimination rules for the symbols for operations of the language of $\mathrm{W}$ - for the system $\mathrm{W}$. We showed that this system W overcomes all the problems pointed out in Wadge's system and it stands up as a proper ND-system, according to all criteria considered in the mentioned literature. 
The data structures of $\mathrm{W}$ are sequents. A sequent of $\mathrm{W}, \mathrm{W}$-sequent, is a pair $\langle\Gamma, \varphi\rangle$, denoted as $\Gamma \Vdash \varphi$, where $\Gamma$ is a finite set of formulas and $\varphi$ is a formula. The tree labels, denoted generically by $L, L_{1}, L_{2}$, are (Ax), (Hp), (0I), (0E), (1I), (1E), (1'I), (1'E), $\left({ }^{-} \mathrm{I}\right),\left({ }^{-} \mathrm{E}\right),(\smile \mathrm{I}),(\smile \mathrm{E}),(+\mathrm{IR}),(+\mathrm{IL}),(+\mathrm{E}),(\cdot \mathrm{I}),(\cdot \mathrm{ER}),(\cdot \mathrm{EL}),(; \mathrm{I})$, and $(; \mathrm{E})$. The proof trees of $\mathrm{W}, \mathrm{W}$-proof trees, are trees whose nodes are $\mathrm{W}$-sequents and whose edges are labelled with tree labels, defined inductively by the following rules of proof tree construction, where $x, y, u, v, w$ are symbols for individuals, $T, U, V$ are $\mathrm{W}$-terms, $\varphi$ is a $\mathrm{W}$-formula, and $\Pi, \Pi_{1}, \Pi_{2}$ are $W$-proof trees:

1. (Ax) The empty tree is a W-proof tree.

2. (Hp) If $\Pi$ is a W-proof tree and $\varphi \in \Gamma$, then $\frac{\Pi}{\Gamma \Vdash \varphi}(\mathrm{Hp})$ is a W-proof tree.

3. (OI) Whenever $\frac{\Pi_{1}}{\Gamma \Vdash x T y}\left(L_{1}\right)$ and $\frac{\Pi_{2}}{\Gamma \Vdash x T^{-} y}\left(L_{2}\right)$ are $\mathrm{W}$-proof trees, then

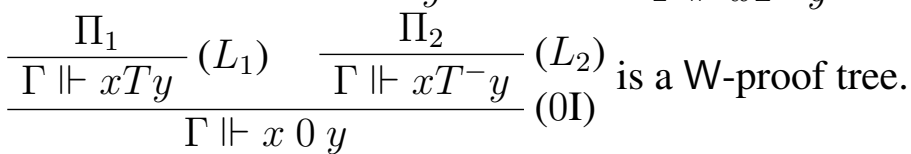

4. (0E) If $\frac{\Pi}{\Gamma \Vdash x 0 y}(L)$ is a W-proof tree, then $\frac{\Pi}{\frac{\Pi \Vdash x 0 y}{\Gamma \Vdash \varphi}(0 \mathrm{E})}(L)$ is a W-proof tree.

5. (1I) If $\Pi$ is a W-proof tree, then $\frac{\Pi}{\Gamma \Vdash x 1 y}$ (1I) is a W-proof tree.

6. (1E) Whenever $\frac{\Pi_{1}}{\Gamma \Vdash x 1 y}\left(L_{1}\right)$ and $\frac{\Pi_{2}}{\Gamma \Vdash u T v}\left(L_{2}\right)$ are $\mathrm{W}$-proof trees, then $\frac{\Pi_{1} \quad \Pi_{2}}{\Gamma \Vdash u T v}(1 \mathrm{E})$ is a W-proof tree.

7. (1'I) If $\Pi$ is a W-proof tree, then $\frac{\Pi}{\Gamma \Vdash x 1^{\prime} x}\left(1^{\prime} \mathrm{I}\right)$ is a W-proof tree.

8. (1'E) Whenever $\frac{\Pi_{1}}{\Gamma \Vdash x T y}\left(L_{1}\right)$ and $\frac{\Pi_{2}}{\Gamma \Vdash u 1^{\prime} y}\left(L_{2}\right)$ are $\mathrm{W}$-proof trees, then

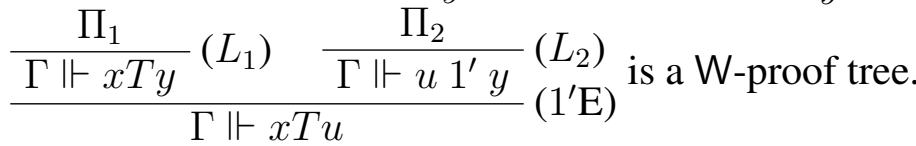

9. $\left({ }^{-} \mathrm{I}\right)$ If $\frac{\Pi}{\Gamma, x T y \Vdash u 0 v}(L)$ is a W-proof tree, then $\frac{\Pi}{\frac{\Gamma, x T y \Vdash u 0 v}{\Gamma \Vdash x T^{-} y}}\left({ }^{-} \mathrm{I}\right)$ is a Wproof tree.

10. ( $\left.{ }^{-} \mathrm{E}\right)$ If $\frac{\Pi}{\Gamma \Vdash x T^{--} y}(L)$ is a W-proof tree, then $\frac{\Pi}{\frac{\Gamma \Vdash x T^{--} y}{\Gamma \Vdash x T y}}\left({ }^{-} \mathrm{E}\right)$ is a W-proof tree.

11. ( $\smile$ I) If $\frac{\Pi}{\Gamma \Vdash x T y}(L)$ is a W-proof tree from $\Gamma$, then $\frac{\frac{\Pi}{\Gamma \Vdash x T y}}{\frac{\Gamma}{\Gamma \Vdash y T}(\llcorner)}(\smile$ I) is a Wproof tree.

12. $(\smile \mathrm{E})$ If $\frac{\Pi}{\Gamma \Vdash x T^{\smile} y}(L)$ is a W-proof, then $\frac{\Pi}{\frac{\Gamma \Vdash x T^{\smile} y}{\Gamma \Vdash y T x}}(L)$ is) is a W-proof tree.

13. $(+\mathrm{IR})$ If $\frac{\Pi}{\Gamma \Vdash x T y}(L)$ is W-proof tree, then $\frac{\frac{\Pi}{\Gamma \Vdash x T y}(L)}{\Gamma \Vdash x T+U y}(+\mathrm{IR})$ is a W-proof tree. 
14. (+IL) If $\frac{\Pi}{\Gamma \Vdash x U y}(L)$ is a W-proof tree, then $\frac{\frac{\Pi}{\Gamma \Vdash x U y}(L)}{\Gamma \Vdash x T+U y}(+\mathrm{IL})$ is a Wproof tree.

15. $(+\mathrm{E})$ If $\frac{\Pi_{1}}{\Gamma \Vdash x T+U y}\left(L_{1}\right), \frac{\Pi_{2}}{\Gamma, x T y \Vdash \varphi}\left(L_{2}\right)$, and $\frac{\Pi_{3}}{\Gamma, x U y \Vdash \varphi}\left(L_{3}\right)$ are Wproof trees, then

$$
\frac{\frac{\Pi_{1}}{\Gamma \Vdash x T+U y}\left(L_{1}\right) \frac{\Pi_{2}}{\Gamma, x T y \Vdash \varphi}\left(L_{2}\right) \frac{\Pi_{3}}{\Gamma, x U y \Vdash \varphi}\left(L_{3}\right)}{\Gamma \Vdash \varphi}(+\mathrm{E})
$$

is a W-proof tree.

16. (.I) Whenever $\frac{\Pi_{1}}{\Gamma \Vdash x T y}\left(L_{1}\right)$ and $\frac{\Pi_{2}}{\Gamma \Vdash x U y}\left(L_{2}\right)$ are $\mathrm{W}$-proof trees, then

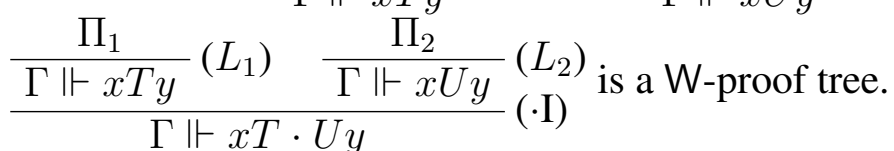

17. (.ER) If $\frac{\Pi}{\Gamma \Vdash x T \cdot U y}(L)$ is a W-proof, then $\frac{\Pi}{\frac{\Gamma \Vdash x T \cdot U y}{\Gamma \Vdash x T y}(\cdot \mathrm{ER})}$ is a W-proof tree.

18. (.EL) If $\frac{\Pi}{\Gamma \Vdash x T \cdot U y}(L)$ is a W-proof, then $\frac{\Pi}{\frac{\Gamma \Vdash x T \cdot U y}{\Gamma \Vdash x U y}(\cdot \mathrm{EL})}(L)$ is a W-proof tree.

19. (;I) Whenever $\frac{\Pi_{1}}{\Gamma \Vdash x T v}\left(L_{1}\right)$ and $\frac{\Pi_{2}}{\Gamma \Vdash v U y}\left(L_{2}\right)$ are $\mathrm{W}$-proof trees, then

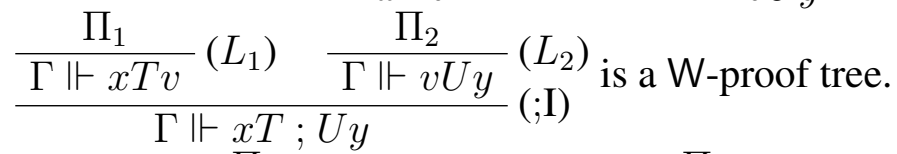

20. (;E) If $\frac{\Pi_{1}}{\Gamma \Vdash x T ; U y}\left(L_{1}\right)$ and $\frac{\Pi_{2}}{\Gamma, x T u, u U y \Vdash \varphi}\left(L_{2}\right)$ are $\mathrm{W}$-proof trees and $u$ does not occur in the formulas of $\Gamma$, then

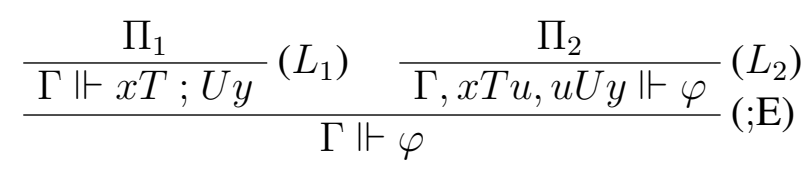

is a W-proof tree.

A proof of a W-sequent $\Gamma \Vdash \varphi$ in W (W-proof of $\Gamma \Vdash \varphi)$, is a W-proof tree $\Pi$, such that $\Gamma \Vdash \varphi$ is the root of $\Pi$. We say that a $W$-formula $\varphi$ is syntactical consequence of a set of formulas $\Gamma$ in W ( $\varphi$ is a W-syntactical consequence of $\Gamma$ ), denoted by $\Gamma \vdash_{W} \varphi$, if there exists a finite $\Gamma^{\prime} \subseteq \Gamma$ and a W-proof of $\Gamma^{\prime} \Vdash \varphi$. We say that a W-formula $\varphi$ is a theorem of $\mathrm{W}$ ( $\varphi$ is a W-theorem) denoted by $\vdash_{\mathrm{W}} \varphi$, if $\varphi$ is W-syntactical consequence of the empty set.

The soundness and completeness of a previous version of $W$ was presented in [Suguitani 2013].

\section{Final comments}

The system W has been successful to frame the Calculus of Binary Relations in a proper ND-system which preserves the behaviour of the algebraic operations on binary relations. 
On the other hand, it misses the algebraic frame of an equational calculus and introduces individual variables in the formulas. As it is well known, the use of individual variables might be highly problematic when considering termination. Besides, this fact can be seen as a disadvantage in relation to Relation Algebra which is developed entirely within the equational environment. Nonetheless, as it was mentioned in Section 1, since Relation Algebra has intrinsic limitations to reach completeness for the standard models of the algebra of binary relations, any alternative for a complete system has to carry the can.

\section{References}

Andréka, H. (1997). Complexity of equations valid in algebras of relations, part i: Strong non-finitizability. Ann. Pure. Appl. Logic, 89:49-209.

De Morgan, A. (1860). On the syllogism, No. IV., and on the logic of relations. Transactions of the Cambridge Philosophical Society, 10:331-358.

Indrzejczak, A. (2010). Natural Deduction, Hybrid Systems and Modal Logics. Springer.

Jónsson, B. (1991). The theory of binary relations. In H. Andréka, D. Monk, I. N., editor, Algebraic Logic. North-Hollan.

Jónsson, B. and Tarski, A. (1948). Representation problems for relation algebras. Bulletin of the American Mathematical Socidety, 54:80.

Lyndon, R. C. (1950). The representation of relational algebras. Annals of Mathematics, 51:707-729.

Lyndon, R. C. (1956). The representation of relational algebras, ii. Annals of Mathematics, 63:294-307.

Maddux, R. D. (1983). A sequent calculus for relation algebras. Ann. Pure Appl. Logic, 25:73-101.

Monk, J. D. (1964). On representable relation algebras. Michigan Mathematical Journal, 11:207-210.

Orlowska, E. (1991). Relational interpretation of modal logics. In H. Andreka, D. Monk, I. N., editor, Algebraic Logic. North-Holland.

Peirce, C. S. (1883). Note B: the logic of relatives. Studies in Logic by Members of the John Hopkins University, pages 187-203.

Schröder, F. W. K. E. (1895). Vorlesungen über die algebra der logik, volume 3, "Algebra und logik de relative", part I. B. G. Teubner, Leipzig.

Suguitani, L. (2013). Sobre a lógica e a aritmética das relações. PhD thesis, UNICAMP.

Sundholm, B. G. (1991). Systems of deduction. In D. Gabbay, F. G., editor, Handbook of Philosophical Logic, Vol. I: Elements of Classical Logic, pages 133-188. D. Reidel.

Tarski, A. (1941). On the calculus of relations. Journal of Symbolic Logic, 6:73-89.

Tarski, A. (1955). Contributions to the theory of models iii. Koninkl. Nederl. Akad. Wetensch, pages 56-64.

Wadge, W. W. (1975). A complete natural deduction system for the relational calculus. Technical report, Department of Computer Science, University of Warwick, Coventry, UK. 